\title{
Interferometric control of nanorotor alignment
}

\author{
Birthe Schrinski, Benjamin A. Stickler, and Klaus Hornberger \\ University of Duisburg-Essen, Faculty of Physics, Lotharstraße 1, 47048 Duisburg, Germany
}

\begin{abstract}
The intrinsically non-linear rotation dynamics of rigid bodies offer unprecedented ways to exploit their quantum motion. In this Letter we devise a rotational analog of Mach-Zehnder interferometry, which allows steering symmetric rotors from fully aligned to completely antialigned. The scheme uses a superposition of four distinct orientations, emerging at the eighth of the quantum revival time, whose interference can be controlled by a weak laser pulse. We develop a semiclassical model of the effect and demonstrate that it persists even in presence of imperfections and decoherence.
\end{abstract}

Introduction. - It is a major aim in the field of optomechanics [1, 2] to control the motion of levitated nanoparticles at the quantum limit [3], as required for fundamental tests and for precision sensing [4 7. Levitated objects have been used in their classical state of motion to search for physics beyond the standard model $[8,9$ and to demonstrate force sensitivities at the zeptonewton level 10, 11. The recent achievement of cooling the center-ofmass motion of a nanosphere to the ground state [12 14 now heralds a new era by mastering the quantum dynamics of internally warm solid objects composed of millions of atoms.

Levitated nanoparticles rotate, adding an intrinsically nonlinear twist to their center-of-mass dynamics. To date, experiments with rotating particles still operate in the classical domain; they spin nanorotors with ultrahigh precision [15, 16] and at ultra-high frequencies [1618], and demonstrate precession [19, 20], radiation-torque heating [21], and record-breaking torque sensitivities [22]. First experimental implementations of rotational cooling [20, 21, 23] suggest that the quantum regime is within reach [24 26] and that even the trapped ground state of the full translational and rotational motion can be prepared [27.

Quantum rotations of molecules and nanoparticles provide unprecedented ways for quantum-enhanced torque sensing and for testing quantum physics [28]. However, it is still an open problem how to steer nanoparticle alignment in free flight, as needed for future sensing and metrology applications. In this Letter, we solve this problem by devising a Mach-Zehnder-type interference scheme operating in the curved and closed manifold of rigid body orientations. The ability to achieve such control over massive objects will enable unforeseen possibilities for orientation-resolved spectroscopy, rotation stateresolved collision and reaction studies, spatially resolved torque and rotation sensing, and quantum superposition tests 28, 29.

The rotational analog of Mach-Zehnder interferometry is based on the phenomenon of orientational quantum revivals, which occur in free rotors as a direct consequence of angular momentum quantization. Specifically, the quadratic dependence of the rotational energies on the total angular momentum quantum number leads to
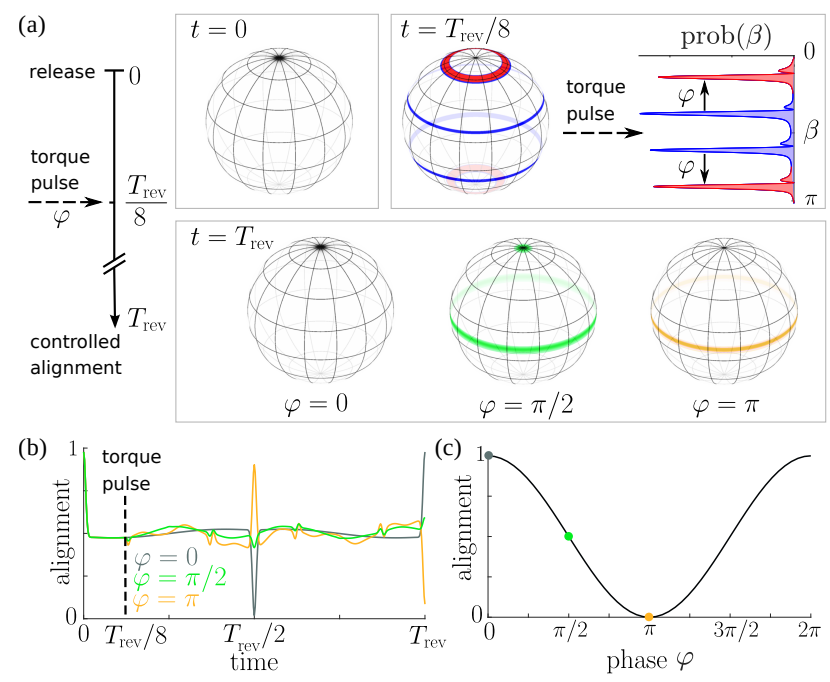

Figure 1. (a) Orientational distribution of the rotor symmetry axis at different times $t$. An initially well-aligned state at the north pole (top left) recurs fractionally as a superposition of wave packets with well-localized latitudes (top right) at $t=T_{\text {rev }} / 8$. A torque pulse imprints a relative phase $\varphi$ onto this superposition even if the interaction cannot distinguish between inverted orientations. Equal phases are indicated by equal colors in the corresponding polar angle distribution $\operatorname{prob}(\beta)$. The torque pulse thus controls whether the state at $t=T_{\mathrm{rev}}$ is fully aligned $(\varphi=0)$, completely antialigned $(\varphi=$ $\pi)$, or a balanced superposition thereof $(\varphi=\pi / 2)$. (b) Time evolution of the corresponding alignment signal $\left\langle\cos ^{2} \beta\right\rangle_{t}$ for $\varphi=0$ (gray), $\varphi=\pi / 2$ (green) and $\varphi=\pi$ (orange). (c) Expected signal $\left\langle\cos ^{2} \beta\right\rangle_{T_{\text {rev }}}$ as a function of $\varphi$.

the recurrence of the initial state [30] at a characteristic revival time $T_{\text {rev }}$, which may be much longer than the wave packet dispersion time. Such revivals have been predicted [31, 32] and observed [33 36] in the alignment of small molecules. Rotational quantum effects have also been proposed for controlling planar and linear rotations of molecules [37, for polarizability metrology [38, for macroscopic quantum superposition tests [39, and for observing the quantum tennis-racket effect [40].

Here we show how the 3D alignment of freely rotating nanoparticles can be controlled by applying a short torque pulse at $T_{\text {rev }} / 8$. It allows tuning the alignment with respect to the initial orientation of the particle 
from fully aligned to completely antialigned, even in the generic case of torques not differing for inverted orientations. This rotational interference scheme makes use of superpositions of well-localized orientational wave packets emerging briefly at fractional revival times. This generalizes coherent rotational control schemes for linear molecules [37] and condensed atom clouds [41 to massive nanoparticles, exhibiting genuine non-commutative $3 \mathrm{D}$ rotations. We show that our scheme works even with millions of rotation states involved, for realistic particle asymmetries, and in presence of environmental decoherence.

Interference scheme. - We first consider symmetric rotors to explain the rotational interference scheme in terms of a semiclassical eight-state model. The free Hamiltonian $\mathrm{H}=\mathrm{J}^{2} / 2 I+\left(1 / 2 I_{c}-1 / 2 I\right) \mathrm{J}_{c}^{2}$ involves the square $\mathrm{J}^{2}$ of the angular momentum vector and its body-fixed component $\mathrm{J}_{c}$, with $I_{c}$ and $I$ the moments of inertia around and orthogonal to the rotor symmetry axis $\mathbf{c}$ [2]. In the free symmetric rotor eigenbasis $|j m k\rangle$ (with $|m|,|k| \leq j$ the quantum numbers for $\mathrm{J}_{z}$ and $\mathrm{J}_{c}$, and $j \in \mathbb{N}_{0}$ the total angular momentum quantum number), the time evolution operator takes the form $\mathrm{U}(t)=\sum_{j m k} e^{-i \pi\left[j(j+1)+\left(I / I_{c}-1\right) k^{2}\right] t / T_{\mathrm{rev}}}|j m k\rangle\langle j m k|$, with $T_{\text {rev }}=2 \pi I / \hbar$. Taking the direction of initial alignment as the space-fixed $z$-axis implies that the angular momentum representation of the aligned initial state $\rho_{0}$ is diagonal in $m$ and $k$. From this it readily follows that any such symmetric rotor state will fully recur, $\mathrm{U}\left(T_{\text {rev }}\right) \rho_{0} \mathrm{U}^{\dagger}\left(T_{\text {rev }}\right)=\rho_{0}$, as a direct consequence of angular momentum quantization.

To characterize fractional revivals, consider a massive prolate rotor $\left(I_{c} \ll I\right)$ in a well-aligned pure initial state, $\rho_{0}=\left|\Psi_{0}\right\rangle\left\langle\Psi_{0}\right|$. Using Euler angles in the $z-y^{\prime}-z^{\prime \prime}$-convention [43], the angle $\beta \in[0 ; \pi]$ between the $z$-axis and the rotor axis is then localized close to $\beta=0$, implying that the quantum numbers of $J_{z}$ and $\mathrm{J}_{c}$ coincide, $\left\langle\alpha, \beta, \gamma \mid \Psi_{0}\right\rangle=\left\langle\beta \mid \psi_{0}\right\rangle \exp \left[i k_{0}(\alpha+\gamma)\right] / 2 \pi$. The total angular momentum quantum number $j$ will be distributed over a wide range of large values so that the Wigner $\mathrm{d}$-matrix elements in $\langle\alpha \beta \gamma \mid j m k\rangle=(j+$ $1 / 2)^{1 / 2} d_{m k}^{j}(\beta) \exp (i m \alpha+i k \gamma) / 2 \pi$ can be replaced by their asymptotic expressions [44] for $|m|,|k| \ll j$,

$$
d_{m k}^{j}(\beta) \simeq \frac{\cos \left[\left(j+\frac{1}{2}\right) \beta+(m-k) \frac{\pi}{2}-\frac{\pi}{4}\right]}{\sqrt{\frac{\pi}{2}\left(j+\frac{1}{2}\right) \sin \beta}} .
$$

We focus first on the time evolution $\left|\psi_{t}\right\rangle=\mathrm{U}_{\beta}\left|\psi_{0}\right\rangle$ for $m=k=0$ and consider finite occupations of $\mathrm{J}_{z}, \mathrm{~J}_{c}$ later on.

The semiclassical propagator for the $\beta$-motion can then be decomposed as

$$
\left\langle\beta\left|\mathrm{U}_{\beta}(t)\right| \beta_{0}\right\rangle \simeq \frac{u_{c}\left(\beta-\beta_{0} ; t\right)+u_{s}\left(\beta+\beta_{0} ; t\right)}{\sqrt{\sin \beta \sin \beta_{0}}}
$$

with

$$
\begin{aligned}
& u_{c}(\beta ; t)=\frac{1}{\pi} \sum_{j=0}^{\infty} e^{-i \pi j(j+1) t / T_{\mathrm{rev}}} \cos \left[\left(j+\frac{1}{2}\right) \beta\right], \\
& u_{s}(\beta ; t)=\frac{1}{\pi} \sum_{j=0}^{\infty} e^{-i \pi j(j+1) t / T_{\mathrm{rev}}} \sin \left[\left(j+\frac{1}{2}\right) \beta\right] .
\end{aligned}
$$

These contributions can be resummed at integer fractions of the revival time by using the expressions

$$
\begin{aligned}
& \sum_{j=0}^{\infty} \cos (j \theta)=\pi \sum_{n=-\infty}^{\infty} \delta(\theta-2 \pi n)+\frac{1}{2} \\
& \sum_{j=0}^{\infty} \sin (j \theta)=\frac{1}{2} \mathcal{P} \cot \left(\frac{\theta}{2}\right)
\end{aligned}
$$

with $\mathcal{P}$ the Cauchy principle value. They hold for smooth $2 \pi$-periodic test functions and can be obtained from the Poisson summation formula.

Specifically, for $t=T_{\text {rev }} / 8$ the summands in (3) can be grouped into four sets of equal phase by splitting the summation index set into residue classes modulo 16 . Using trigonometric addition theorems one finds

$$
\begin{aligned}
& u_{c}\left(\beta ; \frac{T_{\mathrm{rev}}}{8}\right)=\frac{\sqrt{2}}{4} e^{-i 3 \pi / 16} \sum_{n=0}^{3} e^{-i n(n+1) \pi / 8} \\
& \times\left\{\delta\left[\beta-(2 n+1) \frac{\pi}{8}\right]+\delta\left[\beta+(2 n+1) \frac{\pi}{8}\right]\right\}, \\
& u_{s}\left(\beta ; \frac{T_{\mathrm{rev}}}{8}\right)=\frac{1}{\pi} \sin \left(\frac{\beta}{2}\right)+\frac{2}{\pi} \sum_{n=0}^{3} e^{-i n(n+1) \pi / 8} \\
& \times \mathcal{P} \cot (8 \beta)\{\cos [(2 n+1) \beta]-\cos [(15-2 n) \beta]\} .
\end{aligned}
$$

The locations of the delta functions and Cauchy singularities in these expressions imply that a state initially localized at $\beta=0$ will be promoted, at $T_{\text {rev }} / 8$, into a superposition of narrow wave packets localized at $\beta=\pi / 8$, $3 \pi / 8,5 \pi / 8,7 \pi / 8$ due to the constructive interference of all angular momentum states.

The state at $T_{\text {rev }} / 4$ is obtained by applying the propagator (5) twice, or alternatively by resumming the (3) in residue classes modulo 8 . One finds that the initial state is promoted into a superposition of two wave packets localized at $\beta=\pi / 4,3 \pi / 4$. Similarly, after half of the revival time the initial wave packet reappears localized at $\beta=\pi / 2$, implying a perfectly antialigned rotor.

It thus follows from the composition property of $\mathrm{U}_{\beta}(t)$ that the states $\left|\psi_{\ell}\right\rangle=\mathrm{U}_{\beta}\left(\ell T_{\mathrm{rev}} / 8\right)\left|\psi_{0}\right\rangle$ are composed of well-localized wave packets. Denoting by $\left|\xi_{n}\right\rangle$ the wave packet centered at $\beta=n \pi / 8$, the coefficients in $\left|\psi_{\ell}\right\rangle=$ $e^{i \nu_{\ell}} \sum_{n=1}^{7} M_{\ell n}\left|\xi_{n}\right\rangle$ can be read off from the semiclassical propagators $(\ell=1, \ldots, 7)$. They are given by the unitary 
matrix

$$
M_{\ell n}=\frac{1}{2}\left(\begin{array}{ccccccc}
1 & 0 & 1 & 0 & 1 & 0 & 1 \\
0 & \sqrt{2} & 0 & 0 & 0 & \sqrt{2} & 0 \\
1 & 0 & i & 0 & -i & 0 & -1 \\
0 & 0 & 0 & 2 & 0 & 0 & 0 \\
1 & 0 & -1 & 0 & -1 & 0 & 1 \\
0 & \sqrt{2} & 0 & 0 & 0 & -\sqrt{2} & 0 \\
1 & 0 & -i & 0 & i & 0 & -1
\end{array}\right)
$$

and $\nu_{\ell}=(0,0,-\pi / 8,0, \pi / 2, \pi / 4,3 \pi / 8,0)$.

The states $\left|\psi_{0}\right\rangle, \ldots,\left|\psi_{7}\right\rangle$ thus span an eightdimensional subspace. It is well suited for interferometric control of the rotor alignment since phase differences can be imprinted by applying a torque for a brief duration at one or more of the fractional times $\ell T_{\text {rev }} / 8$.

Torque pulses can be realised with an optical pulse polarized in $z$-direction [29]. The interaction energy is then proportional to $\cos ^{2} \beta$ for particles characterised by an optical anisotropy axis, rendering their optical response invariant under the inversion of the orientation $\beta \rightarrow \pi-\beta$. The corresponding phase operator takes the form

$$
\phi=\exp \left(i \sqrt{2} \varphi \cos ^{2} \beta\right) .
$$

It is diagonal in the angle operator $\beta$, with the phase $\varphi$ determined by the electric field $E(t)$ and the particle polarizability anisotropy $\Delta \alpha, \varphi=\Delta \alpha \int d t|E(t)|^{2} / 2 \sqrt{2} \hbar$.

To control the nanoparticle alignment one applies (7) after the rotor has evolved freely for the time $T_{\text {rev }} / 8$. Abbreviating $\tilde{\mathrm{U}} \equiv \mathrm{U}_{\beta}\left(T_{\mathrm{rev}} / 8\right)$, this yields the state

$$
\phi \tilde{U}\left|\psi_{0}\right\rangle=\frac{\left|\xi_{1}\right\rangle+\left|\xi_{7}\right\rangle}{2}+e^{-i \varphi} \frac{\left|\xi_{3}\right\rangle+\left|\xi_{5}\right\rangle}{2}
$$

where we dropped a global phase. It has a relative phase $\varphi$ between wave packets located at arctic and tropic latitudes, see Fig. 1(a). Quantum interference during the free evolution until the revival time transforms this into a superposition of the well-aligned initial state $\left|\psi_{0}\right\rangle$ and the antialigned state $\left|\xi_{4}\right\rangle$,

$$
\tilde{U}^{7} \phi \tilde{U}\left|\psi_{0}\right\rangle=\cos \left(\frac{\varphi}{2}\right)\left|\psi_{0}\right\rangle+\sin \left(\frac{\varphi}{2}\right)\left|\xi_{4}\right\rangle .
$$

By tuning the phase $\varphi$ one can thus control the alignment at the revival time.

This is illustrated in Fig. 1, for an initial state with $\left|\left\langle j \mid \psi_{0}\right\rangle\right|^{2} \propto \exp \left(-j^{2} / 800\right)$. It shows that alignment control is facilitated already at $T_{\text {ev }} / 2$ with the effects of $\varphi=0, \pi$ swapped. Note that the revivals occur only for a short period of time, given by the initial alignment decay, as determined by the angular momentum distribution.

For finite values of $m$ and $k$, it follows from (1) that $u_{\mathrm{c}}(\beta, t)$ remains semiclassically unaffected as long as $|m|,|k| \ll j$, while $u_{\mathrm{s}}(\beta, t)$ only acquires the additional sign $(-)^{m+k}$. Given that the initial state is well aligned, so that $m=k$, the eight-state model and its prediction (9) therefore remain valid for weakly occupied $J_{z}, J_{c}$.

The numerical simulation of realistic particle states requires matrix elements of the phase operator (7) in the angular momentum basis. Since their exact computation gets numerically intractable for large $j$, one can resort to the semiclassical approximation

$$
\left\langle j m k|\phi| j^{\prime} m^{\prime} k^{\prime}\right\rangle=\left.\delta_{m m^{\prime}} \delta_{k k^{\prime}} e^{-i \pi\left|j-j^{\prime}\right| / 4}\left[1+i \sqrt{2 \xi} \frac{32 k^{2} m^{2}}{\left(j+j^{\prime}+1\right)^{4}} \frac{d}{d \xi} \frac{1}{\sqrt{\xi}}\right] e^{-i A_{j+j^{\prime}+1}^{k m} / \xi} J_{\frac{\left|j-j^{\prime}\right|}{2}}\left(\frac{A_{j+j^{\prime}+1}^{k m}}{\xi}\right)\right|_{\xi=1 / \varphi}
$$

with $A_{J}^{k m}=\left(1-4 k^{2} / J^{2}\right)\left(1-4 m^{2} / J^{2}\right) / \sqrt{2}$. It can be obtained from the Bohr-Sommerfeld quantization of the associated action-angle variables [45] in leading order of $m k / j^{2}$, and will be used below.

Alignment control of realistic nanoparticles. - In practice, the initial state will not be perfectly aligned, the rotor will not be completely symmetric, and the quantum dynamics will not be fully coherent. We discuss these imperfections in turn, showing that interferometric alignment control of realistic nanoparticles can still be expected in their presence.

For concreteness, consider an ellipsoidally shaped silicon nanorod with principal diameters of $5.5 \mathrm{~nm}$ and $50 \mathrm{~nm}$, corresponding to a mass of $1.1 \times 10^{6} \mathrm{amu}$ and a revival time of $T_{\text {rev }}=14 \mathrm{~ms}$. After coherent scattering cooling close to the trapped ro-translational ground state in an elliptically polarized tweezer [27, one adiabatically changes to linear polarization such that the intrinsic rotation around the symmetry axis is released with only weakly occupied $J_{c}$. The initial state may then be described by a mixture of $\left\langle\alpha, \beta, \gamma \mid \Psi_{0}\right\rangle \propto \exp \left(-\sin ^{2} \beta / 4 \sigma_{\beta}^{2}+\right.$ $\left.i k_{0}(\alpha+\gamma)\right)$ with $\sigma_{\beta}$ and $k_{0}$ determined by the cooling setup and the adiabatic release. Based on [27] we assume $\sigma_{\beta}=3.1 \times 10^{-3} \mathrm{rad}$ and $k_{0} \in \mathbb{Z}$ distributed as a Gaussian with $\sigma_{k}<6$.

Once the trapping laser is switched off, the particle falls freely, is illuminated by the weak phase pulse at $t=T_{\mathrm{rev}} / 8$ and by a strong readout pulse around $T_{\mathrm{rev}}$, 
before it is recaptured and recooled. The torque needed to imprint the phase $\varphi$ must be applied for a time $\tau$ much shorter than the rotational dispersion time. A pulse with constant amplitude yields $\varphi=\Delta \alpha\left|E_{0}\right|^{2} \tau / \sqrt{2} 4 \hbar$, where $\left|E_{0}\right|^{2}=4 P /\left(\pi w_{0}^{2} \epsilon_{0} c\right)$ depends on the power $P$ and waist $w_{0}$ of the laser beam.

Figure 2(a) shows how the alignment signal at $\varphi=\pi$ is affected by the uncertainty in the initial orientation and by finite values of the intrinsic angular momentum component. Both lead to a moderate reduction of the anti-alignment effect. This is due to the phase operator (7) no longer yielding definite relative phases for wave packets with angular dispersion, and due to the matrix elements 10 effecting an $m$ - and $k$-dependent shift of the revival time. For $\varphi=0$, in contrast, all symmetric rotors display a perfect alignment recurrence.

The asymmetry of a general prolate top is characterized by the parameter $b=\left(I_{a}^{-1}-I_{b}^{-1}\right) /\left(2 I_{c}^{-1}-I_{a}^{-1}-I_{b}^{-1}\right)$, which determines the approximate rotation energies in terms of the symmetric ones [46]. Assuming one of the minor principal diameters to be $5.0 \mathrm{~nm}$ corresponds to $|b|=2.3 \times 10^{-5}$. For such values one may safely approximate the rotational eigenstates by those of a symmetric top, and take into account only the modified rotational energy spectrum.

Figure 2(b) shows the alignment signal at $\varphi=0, \pi$ and the associated shift of the revival time as a function of the asymmetry parameter for the initial state $\left|\Psi_{0}\right\rangle$ (with $\sigma_{\beta}=3 \times 10^{-3} \mathrm{rad}$ and $k_{0}=0$ ). One observes that shape asymmetries may lead to a noticeable reduction of the alignment effect, while only slightly shifting the revival time. However, the asymmetry assumed above, which is well within the capabilities of present-day nanofabrication techniques, still yields a sizable alignment signal of $\left\langle\cos ^{2} \beta\right\rangle=0.87$.

Collisions of the nanorotor with residual gas particles may effect a gradual diffusion of the nanoparticle angular momentum and thus provide a dominant source of decoherence 47 50. Denoting the (operatorvalued) orientation of the symmetry axis by $\mathbf{c}=$ $(\cos \alpha \sin \beta, \sin \alpha \sin \beta, \cos \beta)^{T}$, and neglecting changes in the intrinsic rotation $\mathrm{J}_{c}$, the corresponding master equation reads as $\partial_{t} \rho=-i[\mathrm{H}, \rho] / \hbar+\Gamma_{\text {gas }}(\mathbf{c} \cdot \rho \mathbf{c}-\rho)$, with $\Gamma_{\text {gas }}$ the collision rate [49]. It can be simulated by means of a Monte-Carlo unraveling [51, 52] in terms of the stochastic Schrödinger equation

$$
|\mathrm{d} \Psi\rangle=\frac{1}{i \hbar} \mathrm{H} \mathrm{d} t+\sum_{\ell=1}^{3}\left(\frac{\mathrm{c}_{\ell}}{\sqrt{\left\langle\Psi\left|\mathrm{c}_{\ell}^{2}\right| \Psi\right\rangle}}-1\right)|\Psi\rangle \mathrm{d} N_{\ell}
$$

where the independent Poisson increments $\mathrm{d} N_{\ell}$ have the expectation values $\mathbb{E}\left[\mathrm{d} N_{\ell}\right]=\Gamma_{\text {gas }}\left\langle\Psi\left|\mathrm{c}_{\ell}^{2}\right| \Psi\right\rangle \mathrm{d} t$. Here we used that $\sum_{\ell} \mathrm{c}_{\ell}^{2}=1$, so that $\sum_{\ell} \mathbb{E}\left[\mathrm{d} N_{\ell}\right]=\Gamma_{\text {gas }} \mathrm{d} t$ is state independent.

Figure 2(c) displays the impact of collisional decoherence on the interferometric alignment control for $\Gamma_{\text {gas }}=$
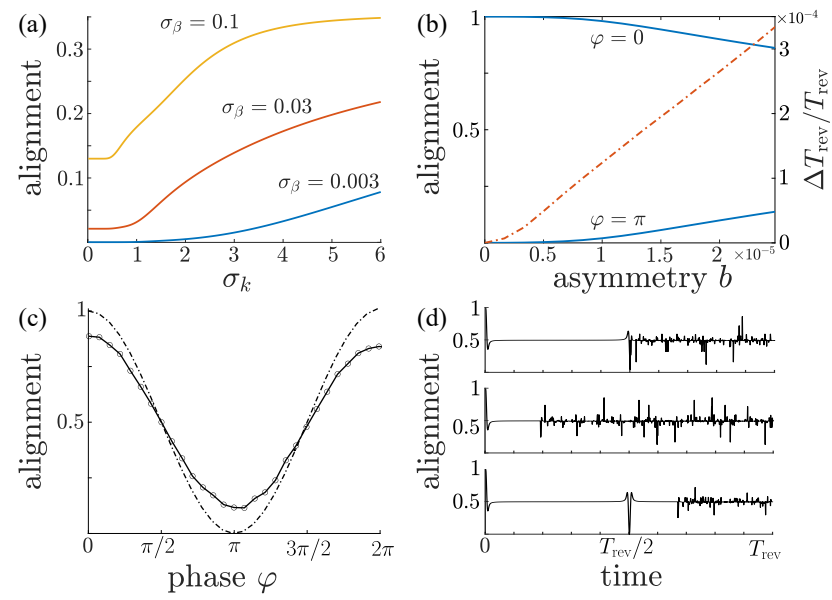

Figure 2. (a) Antialignment effect at $\varphi=\pi$ for realistic initial states with angular spreads $\sigma_{\beta}=0.1, \sigma_{\beta}=0.03$, and $\sigma_{\beta}=0.003$ (top to bottom), as a function of the intrinsic angular momentum width $\sigma_{k}$. For perfectly aligned initial states the signal vanishes identically (9). This interference effect gets impaired for realistic values of $\sigma_{\beta}$ and $\sigma_{k}$, but is still observable. (b) Effect of nanoparticle asymmetry on the alignment for $\varphi=0$ and $\varphi=\pi$. The dash-dotted line shows how the asymmetry increases the revival time (orange, right scale). (c) Collisional decoherence at a nitrogen gas pressure of $5 \times 10^{-9}$ mbar reduces the achievable alignment control (solid line) compared to the case of perfect vacuum (dashdotted line). (d) Alignment associated with three exemplary Monte-Carlo trajectories. (The parameters used for all panels are given in the text, unless specified otherwise.)

$20.7 \mathrm{~Hz}$, corresponding to a nitrogen gas pressure of $5 \times 10^{-9}$ mbar at room temperature. One observes that the interference signal gets slightly degraded for these realistic gas pressures, irrespective of the imprinted phase $\varphi$. For illustration, Fig. 2(d) shows the alignment dynamics of three sample trajectories from the ensemble described by (11).

A further source of decoherence might be the Rayleigh scattering of laser photons from the phase pulse. However, this is negligible since a power of $1.3 \mathrm{~mW}$ suffices to imprint a relative phase of $2 \pi$ (assuming a pulse duration of $t_{\mathrm{L}}=100 \mathrm{~ns}$, a wave length of $1550 \mathrm{~nm}$, and a waist of $\left.w_{0}=30 \mu \mathrm{m}\right)$, implying that only $1.7 \times 10^{-13}$ photons scatter on average during the pulse.

Conclusions. - The interferometric alignment scheme relies only on the free quantum dynamics of symmetric rotors and on their polarization anisotropy. It can thus be employed for a wide range of particle species and sizes, even in absence of internal spin [23, 53], magnetization [54], or dipole moments [55]. The scheme will find applications whenever exquisite control is required of the field-free alignment of single particles in vacuum [29].

We found that the interference effect can be represented in an effective eight-dimensional subspace spanned by superpositions of narrow orientational wave packets. More complex interference schemes are thus conceivable 
by applying several pulses of light at integer multiples of $T_{\text {rev }} / 8$, effecting unitary transitions between these states. They could be used for preparing orientational superposition states, for tests of quantum physics, for precision metrology of molecular properties, or even for processing quantum information [56, 57. Beyond that, it is straightforward to use even smaller integer fractions of the revival time if interference in higher dimensional effective spaces is required.

This work was supported by the Deutsche Forschungsgemeinschaft (DFG-394398290).

[1] M. Aspelmeyer, T. J. Kippenberg, and F. Marquardt, Cavity optomechanics, Rev. Mod. Phys. 86, 1391 (2014)

[2] W. P. Bowen and G. J. Milburn, Quantum optomechanics (CRC press, 2015).

[3] J. Millen, T. S. Monteiro, R. Pettit, and A. N. Vamivakas, Optomechanics with levitated particles, Rep. Prog. Phys. 83, 026401 (2020).

[4] M. Arndt and K. Hornberger, Testing the limits of quantum mechanical superpositions, Nat. Phys. 10, 271 (2014).

[5] J. Millen and B. A. Stickler, Quantum experiments with microscale particles, Contemp. Phys. 61, 155 (2020).

[6] M. Rademacher, J. Millen, and Y. L. Li, Quantum sensing with nanoparticles for gravimetry: when bigger is better, Adv. Opt. Tech. 9, 227 (2020).

[7] D. C. Moore and A. Geraci, Searching for new physics using optically levitated sensors, Quant. Sci. Techn. (2020).

[8] A. D. Rider, D. C. Moore, C. P. Blakemore, M. Louis, M. Lu, and G. Gratta, Search for screened interactions associated with dark energy below the $100 \mu \mathrm{m}$ length scale, Phys. Rev. Lett. 117, 101101 (2016).

[9] F. Monteiro, G. Afek, D. Carney, G. Krnjaic, J. Wang, and D. C. Moore, Search for composite dark matter with optically levitated sensors, Phys. Rev. Lett. 125, 181102 (2020)

[10] G. Ranjit, M. Cunningham, K. Casey, and A. A. Geraci, Zeptonewton force sensing with nanospheres in an optical lattice, Phys. Rev. A 93, 053801 (2016)

[11] D. Hempston, J. Vovrosh, M. Toroš, G. Winstone, M. Rashid, and H. Ulbricht, Force sensing with an optically levitated charged nanoparticle, Appl. Phys. Lett. 111, 133111 (2017).

[12] U. Delić, M. Reisenbauer, K. Dare, D. Grass, V. Vuletić, N. Kiesel, and M. Aspelmeyer, Cooling of a levitated nanoparticle to the motional quantum ground state, Science 367, 892 (2020).

[13] L. Magrini, P. Rosenzweig, C. Bach, A. DeutschmannOlek, S. G. Hofer, S. Hong, N. Kiesel, A. Kugi, and M. Aspelmeyer, Real-time optimal quantum control of mechanical motion at room temperature, Nature 595, 373 (2021).

[14] F. Tebbenjohanns, M. L. Mattana, M. Rossi, M. Frimmer, and L. Novotny, Quantum control of a nanoparticle optically levitated in cryogenic free space, Nature 595, 378-382 (2021).

[15] S. Kuhn, B. A. Stickler, A. Kosloff, F. Patolsky, K. Hornberger, M. Arndt, and J. Millen, Optically driven ultra- stable nanomechanical rotor, Nat. Commun. 8, 1670 (2017).

[16] R. Reimann, M. Doderer, E. Hebestreit, R. Diehl, M. Frimmer, D. Windey, F. Tebbenjohanns, and L. Novotny, $\mathrm{GHz}$ rotation of an optically trapped nanoparticle in vacuum, Phys. Rev. Lett. 121, 033602 (2018)

[17] J. Ahn, Z. Xu, J. Bang, Y.-H. Deng, T. M. Hoang, Q. Han, R.-M. Ma, and T. Li, Optically levitated nanodumbbell torsion balance and $\mathrm{GHz}$ nanomechanical rotor, Phys. Rev. Lett. 121, 033603 (2018).

[18] Y. Jin, J. Yan, S. J. Rahman, J. Li, X. Yu, and J. Zhang, $6 \mathrm{GHz}$ hyperfast rotation of an optically levitated nanoparticle in vacuum, Photonics Res. 9, 1344 (2021).

[19] M. Rashid, M. Toroš, A. Setter, and H. Ulbricht, Precession motion in levitated optomechanics, Phys. Rev. Lett. 121, 253601 (2018).

[20] J. Bang, T. Seberson, P. Ju, J. Ahn, Z. Xu, X. Gao, F. Robicheaux, and T. Li, Five-dimensional cooling and nonlinear dynamics of an optically levitated nanodumbbell, Phys. Rev. Research 2, 043054 (2020).

[21] F. van der Laan, F. Tebbenjohanns, R. Reimann, J. Vijayan, L. Novotny, and M. Frimmer, Sub-kelvin feedback cooling and heating dynamics of an optically levitated librator, Phys. Rev. Lett. 127, 123605 (2021)

[22] J. Ahn, Z. Xu, J. Bang, P. Ju, X. Gao, and T. Li, Ultrasensitive torque detection with an optically levitated nanorotor, Nat. Nanotechn. 15, 89-93 (2020).

[23] T. Delord, P. Huillery, L. Nicolas, and G. Hétet, Spincooling of the motion of a trapped diamond, Nature 580, $56(2020)$

[24] B. A. Stickler, S. Nimmrichter, L. Martinetz, S. Kuhn, M. Arndt, and K. Hornberger, Rotranslational cavity cooling of dielectric rods and disks, Phys. Rev. A 94, 033818 (2016)

[25] T. Seberson and F. Robicheaux, Parametric feedback cooling of rigid body nanodumbbells in levitated optomechanics, Phys. Rev. A 99, 013821 (2019).

[26] F. Tebbenjohanns, A. Militaru, A. Norrman, F. van der Laan, L. Novotny, and M. Frimmer, Optimal orientation detection of an anisotropic dipolar scatterer (2021), arXiv 2109.08444

[27] J. Schäfer, H. Rudolph, K. Hornberger, and B. A. Stickler, Cooling nanorotors by elliptic coherent scattering, Phys. Rev. Lett. 126, 163603 (2021)

[28] B. A. Stickler, K. Hornberger, and M. S. Kim, Quantum rotations of nanoparticles, Nat. Rev. Phys. 3, 589 (2021).

[29] C. P. Koch, M. Lemeshko, and D. Sugny, Quantum control of molecular rotation, Rev. Mod. Phys. 91, 035005 (2019).

[30] R. Robinett, Quantum wave packet revivals, Physics Reports 392, 1 (2004)

[31] T. Seideman, Revival structure of aligned rotational wave packets, Phys. Rev. Lett. 83, 4971 (1999).

[32] M. Machholm and N. E. Henriksen, Field-free orientation of molecules, Phys. Rev. Lett. 87, 193001 (2001)

[33] M. D. Poulsen, E. Péronne, H. Stapelfeldt, C. Z. Bisgaard, S. S. Viftrup, E. Hamilton, and T. Seideman, Nonadiabatic alignment of asymmetric top molecules: Rotational revivals, J. Chem. Phys. 121, 783 (2004).

[34] A. Goban, S. Minemoto, and H. Sakai, Laser-field-free molecular orientation, Phys. Rev. Lett. 101, 013001 (2008) 
[35] O. Ghafur, A. Rouzée, A. Gijsbertsen, W. K. Siu, S. Stolte, and M. J. Vrakking, Impulsive orientation and alignment of quantum-state-selected no molecules, Nat. Phys. 5, 289 (2009).

[36] S. De, I. Znakovskaya, D. Ray, F. Anis, N. G. Johnson, I. A. Bocharova, M. Magrakvelidze, B. D. Esry, C. L. Cocke, I. V. Litvinyuk, and M. F. Kling, Field-free orientation of co molecules by femtosecond two-color laser fields, Phys. Rev. Lett. 103, 153002 (2009).

[37] M. Spanner, E. A. Shapiro, and M. Ivanov, Coherent control of rotational wave-packet dynamics via fractional revivals, Phys. Rev. Lett. 92, 093001 (2004)

[38] J. M. Berglund, M. Drewsen, and C. P. Koch, Femtosecond wavepacket interferometry using the rotational dynamics of a trapped cold molecular ion, New J. Phys. 17, 025007 (2015)

[39] B. A. Stickler, B. Papendell, S. Kuhn, B. Schrinski, J. Millen, M. Arndt, and K. Hornberger, Probing macroscopic quantum superpositions with nanorotors, New J. Phys. 20, 122001 (2018)

[40] Y. Ma, K. E. Khosla, B. A. Stickler, and M. S. Kim, Quantum persistent tennis racket dynamics of nanorotors, Phys. Rev. Lett. 125, 053604 (2020).

[41] F. Kiałka, B. A. Stickler, and K. Hornberger, Orbital angular momentum interference of trapped matter waves, Phys. Rev. Research 2, 022030 (2020)

[42] L. D. Landau and E. Lifshitz, Course of Theoretical Physics Vol 3 Quantum Mechanics (Pergamon Press, 1958).

[43] A. R. Edmonds, Angular momentum in quantum mechanics (Princeton University Press, 1957).

[44] R. G. Littlejohn and L. Yu, Uniform semiclassical approximation for the wigner $6 \mathrm{j}$-symbol in terms of rotation matrices, J. Phys. Chem. A 113, 14904 (2009).

[45] M. S. Child, Semiclassical mechanics with molecular applications (Oxford University Press, 2014).

[46] H. Davis and J. E. Beam, Perturbation formulas for the energy levels of the slightly asymmetric top, J. Molec. Spectrosc. 6, 312 (1961).

[47] B. A. Stickler, B. Papendell, and K. Hornberger, Spatioorientational decoherence of nanoparticles, Phys. Rev. A 94, 033828 (2016).

[48] C. Zhong and F. Robicheaux, Decoherence of rotational degrees of freedom, Phys. Rev. A 94, 052109 (2016)

[49] B. Papendell, B. A. Stickler, and K. Hornberger, Quantum angular momentum diffusion of rigid bodies, New J. Phys. 19, 122001 (2017).

[50] B. A. Stickler, B. Schrinski, and K. Hornberger, Rotational friction and diffusion of quantum rotors, Phys. Rev. Lett. 121, 040401 (2018)

[51] H. Breuer and F. Petruccione, The Theory of Open Quantum Systems (Oxford University Press, 2002).

[52] H. M. Wiseman and G. J. Milburn, Quantum measurement and control (Cambridge University Press, 2009).

[53] T. Delord, L. Nicolas, Y. Chassagneux, and G. Hétet, Strong coupling between a single nitrogen-vacancy spin and the rotational mode of diamonds levitating in an ion trap, Phys. Rev. A 96, 063810 (2017).

[54] J. Gieseler, A. Kabcenell, E. Rosenfeld, J. D. Schaefer, A. Safira, M. J. A. Schuetz, C. Gonzalez-Ballestero, C. C. Rusconi, O. Romero-Isart, and M. D. Lukin, Single-spin magnetomechanics with levitated micromagnets, Phys. Rev. Lett. 124, 163604 (2020)

[55] G. Afek, F. Monteiro, B. Siegel, J. Wang, S. Dickson, J. Recoaro, M. Watts, and D. C. Moore, Control and measurement of electric dipole moments in levitated optomechanics, arXiv preprint arXiv:2108.04406 (2021).

[56] V. V. Albert, J. P. Covey, and J. Preskill, Robust encoding of a qubit in a molecule, Phys. Rev. X 10, 031050 (2020)

[57] A. L. Grimsmo, J. Combes, and B. Q. Baragiola, Quantum computing with rotation-symmetric bosonic codes, Phys. Rev. X 10, 011058 (2020). 